\title{
Abstract
}

\section{Teledermatology and Artificial Intelligence}

\author{
Cristian Navarrete-Dechent, MD \\ Melanoma and Skin Cancer Unit, Department of Dermatology, Escuela de Medicina, Pontificia Universidad Catolica de Chile, Santiago, Chile
}

\section{Corresponding Author:}

Cristian Navarrete-Dechent, MD

Melanoma and Skin Cancer Unit, Department of Dermatology

Escuela de Medicina

Pontificia Universidad Catolica de Chile

Lira 40, Región Metropolitana

Santiago, 8330077

Chile

Phone: 56223542000

Email: ctnavarr@gmail.com

\section{Abstract}

Background: The use of artificial intelligence (AI) algorithms for the diagnosis of skin diseases has shown promise in experimental settings but has not yet been tested in real-life conditions. The COVID-19 pandemic led to a worldwide disruption of health systems, increasing the use of telemedicine. There is an opportunity to include AI algorithms in the teledermatology workflow.

Objective: The aim of this study is to test the performance of and physicians' preferences regarding an AI algorithm during the evaluation of patients via teledermatology.

Methods: We performed a prospective study in 340 cases from 281 patients using patient-taken photos during teledermatology encounters. The photos were evaluated by an AI algorithm and the diagnosis was compared with the clinician's diagnosis. Physicians also reported whether the AI algorithm was useful or not.

Results: The balanced (in-distribution) top-1 accuracy of the algorithm (47.6\%) was comparable to the dermatologists (49.7\%) and residents $(47.7 \%)$ but superior to the general practitioners $(39.7 \% ; P=.049)$. Exposure to the AI algorithm results was considered useful in $11.8 \%$ of visits $(n=40)$ and the teledermatologist correctly modified the real-time diagnosis in $0.6 \%(n=2)$ of cases. Algorithm performance was associated with patient skin type and image quality.

Conclusions: AI algorithms appear to be a promising tool in the triage and evaluation of lesions in patient-taken photographs via telemedicine.

Conflicts of Interest: None declared.

(iproc 2022;8(1):e36894) doi: $10.2196 / 36894$

\section{KEYWORDS}

teledermatology; artificial intelligence; diagnosis; prospective; augmented intelligence; COVID-19

Edited by T Derrick; this is a non-peer-reviewed article. Submitted 28.01.22; accepted 28.01.22; published 09.02.22.

Please cite as:

Navarrete-Dechent $C$

Teledermatology and Artificial Intelligence

iproc 2022;8(1):e36894

URL: https://www.iproc.org/2022/1/e36894

doi: $10.2196 / 36894$

PMID: 
(C) Cristian Navarrete-Dechent. Originally published in Iproceedings (https://www.iproc.org), 09.02.2022. This is an open-access article distributed under the terms of the Creative Commons Attribution License (https://creativecommons.org/licenses/by/4.0/), which permits unrestricted use, distribution, and reproduction in any medium, provided the original work, first published in Iproceedings, is properly cited. The complete bibliographic information, a link to the original publication on https://www.iproc.org/, as well as this copyright and license information must be included. 